\title{
On the strict convexity of the Besicovitch-Orlicz space of almost periodic functions with Orlicz norm
}

\author{
Faculty of Sciences \\ Department of Mathematics \\ University of Tizi-Ouzou \\ Algeria \\ Morsli@ifrance.com \\ fbedouhene@yahoo.fr.
}

Mohamed MorsLI and Fazia Bedouhene

Recibido: 16 de Julio de 2001

Aceptado: 11 de Enero de 2003

\section{ABSTRACT}

The problem of strict convexity of the Besicovitch-Orlicz space of almost periodic functions is considered here in connection with the Orlicz norm. We give necessary and sufficient conditions in terms of the function $\phi$ generating the space.

2000 Mathematics Subject Classification: 46B20, $42 \mathrm{~A} 75$.

Key words: Strict convexity, Besicovitch-Orlicz space, almost periodic functions

\section{Introduction}

The class of Bohr's almost periodic functions denoted by $\{$ u.a.p $\}$ was subject to different generalizations. In [1], A.S. Besicovitch considered this class in the context of the Lebesgue $L^{p}$-spaces. Later, T.R. Hillmann [3] extended the work [1] to the context of Orlicz spaces, defining the large class of Besicovitch-Orlicz almost periodic functions.

All this work concerned different aspects of structure and topological properties. In [5], [6], [7], we characterized the strict and uniform convexity of this space equipped with the Luxemburg norm.

In this note, we consider the Orlicz norm case and give a characterization of the strict convexity of this space in terms of regularity conditions on the generating function $\phi$.

The results are of the same kind as those known in the classical Orlicz spaces. The methods are however different in many parts since the Orlicz case arguments are not valid in our space. We developed specific technical results in this sense. 


\section{Preliminaries}

\subsection{Orlicz functions}

Let $\phi: \mathbb{R} \rightarrow \mathbb{R}^{+}$be an Orlicz function, i.e. it satisfies the following conditions: $\phi$ is even, convex, $\phi(u)=0$ iff $u=0$ and $\lim _{u \rightarrow 0} \frac{\phi(u)}{u}=0, \lim _{u \rightarrow \infty} \frac{\phi(u)}{u}=+\infty$.

The function $\phi$ is said to satisfy the $\Delta_{2}$-condition when, there exist $K>2$ and $u_{0} \geq 0$ such that

$$
\phi(2 u) \leq K \phi(u), \quad \forall u \geq u_{0}
$$

An Orlicz function admits, except on a denumerable set, a derivative $\varphi: \mathbb{R}^{+} \rightarrow \mathbb{R}^{+}$ which satisfies $\varphi(0)=0, \varphi(|u|)>0$ if $u \neq 0$, and $\lim _{|u| \rightarrow \infty} \varphi(|u|)=+\infty$.

The derivative $\varphi$ is increasing to infinity and moreover (c.f.[8]),

$$
u \varphi(u) \leq \phi(2 u) \leq 2 u \varphi(2 u), \forall u \geq 0
$$

From [8], we know that if $\phi$ is an Orlicz function then, for each $\varepsilon>0$ there exists an equivalent Orlicz function $\phi_{1}$ with a continuous derivative, such that

$$
(1-\varepsilon) \phi(x) \leq \phi_{1}(x) \leq \phi(x), \forall x \geq 0 .
$$

The function $\psi(y)=\sup \{x|y|-\phi(x), x \geq 0\}$ is called conjugate to $\phi$, it is an Orlicz function when $\phi$ is. The pair $(\phi, \psi)$ satisfies the Young's inequality:

$$
x y \leq \phi(x)+\psi(y), \quad x, y \in \mathbb{R},
$$

Let now $\phi$ be strictly convex, then (c.f.[2]) : for every $k>0$, and $\varepsilon>0$, there exists $\delta>0$ such that

$$
\phi\left(\frac{u+v}{2}\right) \leq(1-\delta) \frac{\phi(u)+\phi(v)}{2}
$$

for all $u, v \in \mathbb{R}$ satisfying $|u| \leq k,|v| \leq k$ and $|u-v| \geq \varepsilon$.

\subsection{The Besicovitch-Orlicz space of almost periodic functions}

Let $M(\mathbb{R})$ denote the linear set of Lebesgue measurable functions on $R$. The functional,

$$
\rho_{B^{\phi}}: M(\mathbb{R}) \rightarrow[0,+\infty], \rho_{B^{\phi}}(f)=\varlimsup_{T \rightarrow+\infty} \frac{1}{2 T} \int_{-T}^{+T} \phi(|f(t)|) d t
$$

is a pseudomodular (c.f.[3], [7]).

The associated modular space, 


$$
B^{\phi}(\mathbb{R})=\left\{f \in M(\mathbb{R}), \lim _{\alpha \rightarrow 0} \rho_{B^{\phi}}(\alpha f)=0\right\}
$$

is called the Besicovitch-Orlicz space (c.f.[3], [8]).

This space is usually equipped with the Luxemburg pseudonorm (c.f.[3], [8]),

$$
\|f\|_{B^{\phi}}=\inf \left\{k>0, \rho_{B^{\phi}}\left(\frac{f}{k}\right) \leq 1\right\}, f \in B^{\phi}(\mathbb{R})
$$

Let $\mathcal{P}$ be the linear set of all generalized trigonometric polynomials, i.e.;

$$
\mathcal{P}=\left\{P(t)=\sum_{k=1}^{n} \alpha_{k} \exp \left(i \lambda_{k} t\right), \lambda_{k} \in \mathbb{R}, \alpha_{k} \in \mathbb{C}, k \in \mathbb{N}\right\},
$$

The Besicovitch-Orlicz space of almost periodic functions denoted by $B^{\phi} a . p$. is the closure of $\mathcal{P}$ in $B^{\phi}(\mathbb{R})$ with respect to the pseudonorm $\|\cdot\|_{B^{\phi}}$ :

$$
B^{\phi} \text { a.p. }=\left\{f \in B^{\phi}(\mathbb{R}), \exists p_{n} \in \mathcal{P}, n=1,2, \ldots ; \text { s.t. } \lim _{n \rightarrow \infty}\left\|f-p_{n}\right\|_{B^{\phi}}=0\right\}
$$

From [3], [8], we know that $\phi(|f|) \in B^{1}$ a.p. when $f \in B^{\phi}$ a.p. and from a classical result in [1] we deduce the existence of the limit in the expression of $\rho_{B^{\phi}}(f)$, i.e. :

$$
\rho_{B^{\phi}}(f)=\lim _{T \rightarrow+\infty} \frac{1}{2 T} \int_{-T}^{+T} \phi(|f(t)|) d t, f \in B^{\phi} a . p .
$$

We denote by $\{$ u.a.p $\}$ the classical algebra of Bohr's almost periodic functions or, what is the same, the closure of $\mathcal{P}$ in the uniform metric. We know that $\phi(|f|) \in$ $\{$ u.a.p $\}$ when $f \in\{$ u.a.p $\}$ (c.f.[1]).

Furthermore, from [1] we know that $M(|f|)>0$ if $f \in\{$ u.a.p $\}, f \neq 0$, where

$$
M(f)=\lim _{T \rightarrow+\infty} \frac{1}{2 T} \int_{-T}^{+T} f(t) d t .
$$

For each $f \in B^{\phi}$ a.p., we define the Bohr's transform of $f, a(\lambda, f)=M(f \exp (i \lambda t))$, $\lambda \in \mathbb{R}$. There is at most a denumerable set $\left\{\lambda_{1}, \lambda_{2}, \ldots, \lambda_{n}, \ldots\right\}$ of values of $\lambda \in \mathbb{R}$ for which $a(\lambda, f) \neq 0,(c . f .[1],[3],[8])$.

Questions concerning the convergence of the formal Fourier series

$$
S(f)(x)=\sum_{n \geq 1} a\left(\lambda_{n}, f\right) \exp \left(i \lambda_{n} x\right)
$$

are not trivial and only partial results are available. 
The Bochner approximation result will be of importance in the sequel :

For $f \in B^{\phi}$ a.p., there exists a sequence $\left\{\sigma_{m}(f)\right\}, m \geq 1$ of generalized trigonometric polynomials ( the Bochner-Fejer's approximation polynomials ) of the form,

$$
\sigma_{m}(f)(x)=\sum_{k=1}^{r_{m}} \mu_{m_{k}} a\left(\lambda_{k}, f\right) \exp \left(i \lambda_{k} x\right), \quad(c . f .[1],[3],[7])
$$

where the convergence factors $\left\{\mu_{m_{k}}\right\}$ depend only on the exponents $\lambda_{k}, k \geq 1$ of the function $f$ and are such that $0<\mu_{m_{k}} \leq 1$.

The sequence $\left\{\sigma_{m}(f)\right\}$ satisfies moreover these fundamental approximation properties (c.f.[3], [7]) :

(1) $\left\|\sigma_{m}(f)\right\|_{B^{\phi}} \leq\|f\|_{B^{\phi}}, m=1,2, \ldots \quad\left(\right.$ and $\left.\rho_{B^{\phi}}\left(\sigma_{m}(f)\right) \leq \rho_{B^{\phi}}(f)\right)$,

$(2)\left\|\sigma_{m}(f)-f\right\|_{B^{\phi}} \rightarrow 0$ when $m \rightarrow \infty \quad\left(\forall \alpha>0, \rho_{B^{\phi}}\left(\alpha\left(\sigma_{m}(f)-f\right)\right) \rightarrow 0\right.$ as $\left.m \rightarrow \infty\right)$.

Let us mention that $\|\cdot\|_{B^{\phi}}$ is in fact a norm on $\{u \cdot a . p\}$.

To end this section, as usual in such spaces, one may define in the space $B^{\phi} a . p$. the following Orlicz pseudonorm,

$$
\|\| f \|_{B^{\phi}}=\sup \left\{M(|f g|), g \in B^{\psi} \text { a.p., } \rho_{B^{\psi}}(g) \leq 1\right\} .
$$

\section{Auxiliary results}

A sequence $\left\{f_{k}\right\}_{k \geq 1}, f_{k} \in B^{\phi}(\mathbb{R})$ is called modular convergent to $f \in B^{\phi}(\mathbb{R})$, when $\lim _{k \rightarrow \infty} \rho_{B^{\phi}}\left(f_{k}-f\right) \stackrel{=0}{=}$.

Let $\Sigma(\mathbb{R})$ be the $\Sigma$-algebra of Lebesgue measurable subsets of $\mathbb{R}$. We define the set function

$$
\bar{\mu}(A)=\varlimsup_{T \rightarrow \infty} \frac{1}{2 T} \int_{-T}^{+T} \chi_{A}(t) d t=\varlimsup_{T \rightarrow \infty} \frac{1}{2 T} \mu([-T,+T] \cap A)
$$

where $\chi_{A}$ denotes the characteristic set function of $A \in \Sigma(R)$.

Clearly, $\bar{\mu}$ is null on sets with $\mu$-finite measure. Moreover, $\bar{\mu}$ is not $\sigma$-additive.

As usual, a sequence of $\Sigma$-measurable functions $\left\{f_{k}\right\}_{k>1}$ is called $\bar{\mu}$ - convergent to $f$ when, for all $\varepsilon>0$,

$$
\lim _{k \rightarrow \infty} \bar{\mu}\left\{t \in \mathbb{R},\left|f_{k}(t)-f(t)\right| \geq \varepsilon\right\}=0 .
$$

From easy computations we can show the following

$$
\forall \varepsilon>0, \exists \delta>0, \forall A \in \Sigma, \bar{\mu}(A) \geq \varepsilon \Rightarrow\left\|\chi_{A}\right\|_{B^{\phi}}>\delta .
$$


Let now $\left\{A_{i}\right\}_{i \geq 1}, A_{i} \in \Sigma$ be such that $A_{i} \cap A_{j}=\emptyset$ if $i \neq j$ and $\bigcup_{i \geq 1} A_{i} \subset[0, \alpha], \alpha<$ 1 .

Put $f=\sum_{i \geq 1} a_{i} \chi_{A_{i}}$ with $\sum_{i \geq 1} \phi\left(a_{i}\right) \mu\left(A_{i}\right)<+\infty$ and let $\tilde{f}$ be the periodic extension of $f$ to the whole $\mathbb{R}$ (with period $\tau=1$ ). Then, there exists a sequence $\left\{P_{m}\right\}_{m \geq 1}, P_{m} \in \mathcal{P}$ such that

$$
\left.\rho_{B^{\phi}}\left(\frac{\tilde{f}-P_{m}}{4}\right) \rightarrow 0 \text { as } m \rightarrow+\infty \text {. (c.f. }[5]\right)
$$

Now, we state some technical results that will be used in the sequel :

Proposition 3.1. (c.f.[5], [6], [7])

Let $\left\{f_{k}\right\}_{k \geq 1}$ be a sequence of functions from $B^{\phi}(\mathbb{R})$. We have the following :

(1) If $f \in B^{\phi}(\mathbb{R})$ is such that $\lim _{k \rightarrow \infty} \rho_{B^{\phi}}\left(f_{k}-f\right)=0$ and there exists $g \in B^{\phi}$.a.p. for which $\max \left(\left|f_{k}\right|,|f|\right) \leq g$, then $\lim _{k \rightarrow \infty} \rho_{B^{\phi}}\left(f_{k}\right)=\rho_{B^{\phi}}(f)$.

(2) If $f \in B^{\phi}$.a.p. and $\left\{P_{n}\right\}$ is the sequence of Bochner-Fejer's approximation polynomials associated to $f$ we have, $\lim _{n \rightarrow \infty} \rho_{B^{\phi}}\left(P_{n}\right)=\rho_{B^{\phi}}(f)$ and $\lim _{n \rightarrow \infty}\left\|P_{n}\right\|_{B^{\phi}}=$ $\|f\|_{B^{\phi}}$

(3) If $f \in B^{\phi}$.a.p. is such that $\lim _{n \rightarrow \infty} \rho_{B^{\phi}}\left(f_{n}-f\right)=0$, then
(a) $\varliminf_{k \rightarrow \infty} \rho_{B^{\phi}}\left(f_{k}\right) \geq \rho(f)$.
(b) $\left\{f_{k}\right\}_{k \geq 1}$ is $\bar{\mu}$-convergent to $f$.

Proposition 3.2. Let $f \in B^{\phi}$.a.p. with $\|f\|_{B^{\phi}}=1$. Then the following conditions hold true :

(1) There exist real numbers $0<\alpha<\beta$ and $\theta \in] 0,1[$ such that for the set $G=$ $\{t \in \mathbb{R}, \alpha \leq|f(t)| \leq \beta\}$ we have $\bar{\mu}(G) \geq \theta$

(2) If $\left\{f_{n}\right\}_{n \geq 1}, f_{n} \in B^{\phi}$.a.p. is a sequence modular convergent to $f$, we have $\bar{\mu}\left(G_{n}\right) \geq$ $\theta_{1}, \quad \forall n \geq n_{1}$, where $G_{n}=\left\{t \in \mathbb{R}, \alpha_{1} \leq\left|f_{n}(t)\right| \leq \beta_{1}\right\}$ and $\alpha_{1}=\frac{\alpha}{2}, \beta_{1}=$ $\frac{\alpha}{2}+\beta, \theta_{1}=\frac{\theta}{2}$, with $\alpha, \beta$ and $\theta$ from part 1 .

\section{Proof.}


(1) The proof will consist of two steps.

Let us show first that taking $\bar{\theta} \in] 0,1\left[\right.$ there exist $\beta>0$ and $T_{0}>0$ such that for all $T \geq T_{0}$ there holds :

$$
\mu(\bar{G} \cap[-T, T]) \geq \bar{\theta} .2 T \text { where } \bar{G}=\{t \in \mathbb{R},|f(t)| \leq \beta\}
$$

For, let $\bar{\theta} \in] 0,1[$, take $\beta>0$ such that $\phi(\beta) .(1-\bar{\theta}) \geq 2$. Supposing that (3.2) is not true, there will exist a sequence $\left(T_{n}\right)_{n \geq 1}$, increasing to infinity for which $\mu\left(\bar{G} \cap\left[-T_{n}, T_{n}\right]\right) \leq \bar{\theta} .2 T_{n}$ and thus,

$$
\begin{aligned}
\frac{1}{2 T_{n}} \int_{-T_{n}}^{T_{n}} \phi(|f|) d t & =\frac{1}{2 T_{n}} \int_{\left(\bar{G} \cap\left[-T_{n}, T_{n}\right]\right)} \phi(|f|) d t+\frac{1}{2 T_{n}} \int_{\bar{G}^{c} \cap\left[-T_{n}, T_{n}\right]} \phi(|f|) d t \\
& \geq \frac{1}{2 T_{n}} \int_{\bar{G}^{c} \cap\left[-T_{n}, T_{n}\right]} \phi(|f|) d t \\
& \geq \frac{1}{2 T_{n}} \phi(\beta) \cdot(1-\bar{\theta}) \cdot 2 T_{n} \\
& \geq \phi(\beta) \cdot(1-\bar{\theta}) \geq 2
\end{aligned}
$$

(Here the notation $\bar{G}^{c}$ is used for the complementary of $\bar{G}$ ).

Finally, since the limit $\lim _{n \rightarrow+\infty} \frac{1}{2 T_{n}} \int_{-T_{n}}^{T_{n}} \phi(|f|) d t$ exists and is finite, we will get $\rho_{B^{\phi}}(f) \geq 2$. A contradiction.

Let now $\delta \in] 0,1[$ then, there exist $\widetilde{\theta} \in] 0,1\left[\right.$ and $T_{0}>0$ such that

$$
\mu(\widetilde{G} \cap[-T, T]) \leq \widetilde{\theta} \cdot 2 T \text { for } T \geq T_{0},
$$

where $\widetilde{G}=\left\{t \in \mathbb{R},|f(t)| \leq \phi^{-1}(1-\delta)\right\}$.

For, let $\left\{P_{n}\right\}_{n>1}$ be the sequence of generalized trigonometric polynomials that converge to $f$ i.e., $\left\|f-P_{n}\right\|_{B^{\phi}} \rightarrow 0$, when $n \rightarrow \infty$. Take $P_{\delta}$ such that $\rho_{B^{\phi}}\left(2\left(f-P_{\delta}\right)\right)<\frac{\delta}{2}$. Since $P_{\delta}$ is uniformly bounded, put $M=\sup _{\mathbb{R}} \phi\left(2\left|P_{\delta}(t)\right|\right)$ and let $\varepsilon>0$ be such that $\frac{\delta}{2}+M \varepsilon<\delta$. Suppose now that (3.3) is not true. Taking $\widetilde{\theta}=1-\varepsilon$, there exists a sequence $\left\{T_{n}\right\}_{n \geq 1}$ increasing to infinity for 
which $\mu\left(\widetilde{G} \cap\left[-T_{n}, T_{n}\right]\right)>\widetilde{\theta} \cdot 2 T_{n}, \forall n \geq n_{0}$ and then,

$$
\begin{aligned}
\frac{1}{2 T_{n}} \int_{-T_{n}}^{T_{n}} \phi(|f|) d t & =\frac{1}{2 T_{n}} \int_{\left(\widetilde{G} \cap\left[-T_{n}, T_{n}\right]\right)} \phi(|f|) d t+\frac{1}{2 T_{n}} \int_{\widetilde{G}^{c} \cap\left[-T_{n}, T_{n}\right]} \phi(|f|) d t \\
& \leq(1-\delta)+\frac{1}{2 T_{n}} \int_{\widetilde{G}^{c} \cap\left[-T_{n}, T_{n}\right]} \phi(|f|) d t
\end{aligned}
$$

and since,

$$
\begin{aligned}
& \frac{1}{2 T_{n}} \int_{\widetilde{G}^{c} \cap\left[-T_{n}, T_{n}\right]} \phi(|f|) d t \\
\leq & \frac{1}{2}\left\{\frac{1}{2 T_{n}} \int_{\widetilde{G}^{c} \cap\left[-T_{n}, T_{n}\right]} \phi\left(2\left|f-P_{\delta}\right|\right) d t+\frac{1}{2 T_{n}} \int_{\widetilde{G}^{c} \cap\left[-T_{n}, T_{n}\right]} \phi\left(2\left|P_{\delta}\right|\right) d t\right\} \\
\leq & \frac{1}{2}\left[\frac{\delta}{2}+M \varepsilon\right] \leq \frac{\delta}{2} .
\end{aligned}
$$

we get finally $\frac{1}{2 T_{n}} \int_{-T_{n}}^{T_{n}} \phi(|f|) d t \leq 1-\frac{\delta}{2}$ and then $\rho_{B^{\phi}}(f) \leq 1-\frac{\delta}{2}$, a contradiction with $\rho_{B^{\phi}}(f)=1$.

We now show the result 1 of the proposition. Let $\delta \in] 0,1\left[\right.$, put $\alpha=\phi^{-1}(1-\delta)$ and consider the set $G=\{t \in \mathbb{R}, \alpha \leq|f(t)| \leq \beta\}$ where $\beta$ is the constant from (3.2) more precisely :

Choose $\widetilde{\theta}$ as in (3.3) then take $\bar{\theta}>\widetilde{\theta}$ as in (3.2) and finally $\beta>\alpha$ as in (3.2). We get,

$$
\begin{aligned}
G^{c} \cap[-T, T]= & \{t \in[-T, T],|f(t)| \leq \alpha\} \cup\{t \in[-T, T],|f(t)| \geq \beta\} \\
& (\widetilde{G} \cap[-T, T]) \cup\left(\bar{G}^{c} \cap[-T, T]\right)
\end{aligned}
$$

and then,

$$
\begin{aligned}
\mu\left(G^{c} \cap[-T, T]\right) & \leq \mu(\widetilde{G} \cap[-T, T])+\mu\left(\bar{G}^{c} \cap[-T, T]\right) \leq \widetilde{\theta} \cdot 2 T+(1-\bar{\theta}) \cdot 2 T \\
& \leq(1-\bar{\theta}+\widetilde{\theta}) \cdot 2 T
\end{aligned}
$$

Finally $\mu(G \cap[-T, T]) \geq(\bar{\theta}-\widetilde{\theta}) 2 T$ for $T \geq T_{0}$ and the proof of 1 is finished. 
(2) Take $\alpha_{1}=\frac{\alpha}{2}, \beta_{1}=\frac{\alpha}{2}+\beta$ and $\theta_{1}=\frac{\theta}{2}$ then, since $\left\{f_{n}\right\}_{n \geq 1}$ is modular convergent to $f$ it is also $\bar{\mu}-$ convergent to $f$ (see 3 of proposition 3.1), it follows then

$$
\bar{\mu}\left\{t \in \mathbb{R},\left|f_{n}(t)-f(t)\right| \geq \frac{\alpha}{2}\right\}<\frac{\theta}{2}, \text { for } n \geq n_{0} .
$$

Putting $F_{n}=\left\{t \in \mathbb{R},\left|f_{n}(t)-f(t)\right| \geq \frac{\alpha}{2}\right\}$, we will have $G \backslash F_{n} \subset G_{n}, \forall n \geq n_{0}$. Indeed, if $t \in G \backslash F_{n}$ then $\alpha \leq|f(t)| \leq \beta$ and $\left|f_{n}(t)-f(t)\right| \leq \frac{\alpha}{2}$. Moreover, from the inequalities, $|f(t)|-\left|f_{n}(t)-f(t)\right| \leq\left|f_{n}(t)\right| \leq\left|f_{n}(t)-f(t)\right|+|f(t)|$ we get, $\alpha_{1} \leq\left|f_{n}(t)\right| \leq \beta_{1}, \forall n \geq n_{0}$, and thus $t \in G_{n}, \forall n \geq n_{0}$.

Finally,

$$
\forall n \geq n_{0}, \bar{\mu}\left(G_{n}\right) \geq \bar{\mu}\left(G \backslash F_{n}\right) \geq \bar{\mu}(G)-\bar{\mu}\left(F_{n}\right) \geq \theta-\frac{\theta}{2}=\theta_{1} .
$$

The assertion follows immediately.

Lemma 3.3. Let $f \in B^{\phi}$ a.p. and $E \in \Sigma$. Then, the function

$$
F:] 0,+\infty\left[\rightarrow \mathbb{R}, F(\lambda)=\rho_{\phi}\left(\frac{f \chi_{E}}{\lambda}\right)\right.
$$

is continuous on $] 0,+\infty[$.

Proof. Let $\lambda_{0}>0$ and $\left\{\lambda_{n}\right\}$ be a sequence of scalars such that $\lim _{n \rightarrow \infty} \lambda_{n}=\lambda_{0}$. We have

$$
\rho_{B^{\phi}}\left[\left(\frac{1}{\lambda_{n}}-\frac{1}{\lambda_{0}}\right) f \chi_{E}\right] \leq\left|\frac{1}{\lambda_{n}}-\frac{1}{\lambda_{0}}\right| \rho_{B^{\phi}}\left(f \chi_{E}\right) \rightarrow 0 \text { when } n \rightarrow \infty .
$$

It follows that $\left\{\frac{1}{\lambda_{n}} f \chi_{E}\right\}$ is modular convergent to $\frac{1}{\lambda_{0}} f \chi_{E}$.

Moreover, we have

$$
\max \left(\frac{1}{\left|\lambda_{n}\right|}|f| \chi_{E}, \frac{1}{\left|\lambda_{0}\right|}|f| \chi_{E}\right) \leq A|f| \in B^{\phi} \text { a.p. }
$$

for some constant $A>0$.

From lemma 3.1, it follows directly that

$$
\lim _{n \rightarrow \infty} \rho_{B^{\phi}}\left(\frac{f \chi_{E}}{\lambda_{n}}\right)=\rho_{B^{\phi}}\left(\frac{f \chi_{E}}{\lambda_{0}}\right) .
$$

Which means that $F$ is continuous at $\lambda_{0}$

Proposition 3.4. Let $f \in B^{\phi}$ a.p., $\|f\|_{B^{\phi}} \neq 0$ then, 
(1) $\||f|\|_{B^{\phi}}=\inf \left\{\frac{1}{k}\left(1+\rho_{B^{\phi}}(k f)\right), k>0\right\}$.

Moreover, there exists $k_{0} \in K(f)=\left\{k>0,\||f|\|_{B^{\phi}}=\frac{1}{k}\left(1+\rho_{B^{\phi}}(k f)\right)\right\}$.

(2) $\rho_{B^{\phi}}\left(\frac{f}{\||f|\|_{B^{\phi}}}\right) \leq 1$,

(3) $\|f\|_{B^{\phi}} \leq\||f|\|_{B^{\phi}} \leq 2\|f\|_{B^{\phi}}$.

Proof. Let us note that by arguments similar to those used in the Orlicz space case, we may show that,

$$
\||f|\|_{B^{\phi}} \leq 2\|f\|_{B^{\phi}} .
$$

(1) From the Young's inequality we have

$$
M(|f g|)=\frac{1}{k} M(|k f g|) \leq \frac{1}{k}\left[\rho_{B^{\phi}}(k f)+\rho_{B^{\psi}}(g)\right], k>0 .
$$

and then,

$$
\||f|\|_{B^{\phi}} \leq \inf _{k>0}\left\{\frac{1}{k}\left(1+\rho_{B^{\phi}}(k f)\right)\right\}
$$

For the opposite inequality, we proceed in several steps :

We suppose first that the derivative $\varphi$ of $\phi$ is continuous.

Let $P \in \mathcal{P}$, then there exists $\left.k_{0} \in\right] 0,+\infty[$ such that,

$$
\||P|\|_{B^{\phi}}=\frac{1}{k_{0}}\left(1+\rho_{B^{\phi}}\left(k_{0} P\right)\right)
$$

For, define the function $F:\left[0,+\infty\left[\rightarrow\left[0,+\infty\left[, F(k)=\rho_{B \psi}[\varphi(k|P(t)|)]\right.\right.\right.\right.$, we claim that $\lim _{k \rightarrow \infty} F(k)=+\infty$. Indeed, if $P \neq 0$, from proposition 3.2, there exist $\alpha, \beta, \theta$, with $\beta>\alpha>0$ and $\theta \in(0,1)$ such that $\bar{\mu}(G) \geq \theta$ where $G=$ $\{t \in \mathbb{R}, \alpha \leq|P(t)| \leq \beta\}$. It follows then,

$$
\rho_{B^{\psi}}[\varphi(k|P|)] \geq \varlimsup_{T \rightarrow+\infty} \frac{1}{2 T} \int_{[-T,+T] \cap G} \psi(\varphi(k|P(x)|)) d x \geq \theta . \psi[\varphi(k \alpha)] .
$$

Then, since an Orlicz function increases to infinity with it's derivative (c.f.[2], [8]), we get $\lim _{k \rightarrow \infty} F(k)=+\infty$.

We now show that $F$ is continuous. For, let $\left.k_{0} \in\right] 0,+\infty\left[\right.$ and $\left\{k_{n}\right\}$ be a sequence of scalars converging to $k_{0}$. Since a trigonometric polynomials is uniformly bounded, we put $\|P\|_{\infty}=M$. Let $\varepsilon>0$ be arbitrary, since $\varphi$ is uniformly continuous on the interval $\left[\frac{k_{0}}{2}, \frac{3 k_{0}}{2}\right]$ there exists $n_{0}$ such that $n \geq n_{0} \Rightarrow$ $\left|\varphi\left(k_{n}|P|\right)-\varphi\left(k_{0}|P|\right)\right| \leq \psi^{-1}(\varepsilon)$ and then

$$
\rho_{B^{\psi}}\left[\varphi\left(k_{n}|P|\right)-\varphi\left(k_{0}|P|\right)\right] \leq \varepsilon .
$$


Let us put $f_{n}=\varphi\left(k_{n}|P|\right)$ and $f=\varphi\left(k_{0}|P|\right)$ then $f_{n} \in\{$ u.a.p. $\}$ and $f \in$ $\left\{\right.$ u.a.p.\}. Since $\varphi$ is increasing, we have moreover $f_{n} \leq \varphi\left(2 k_{0}|P|\right)$. Now, from (3.6) we have $\lim _{n \rightarrow \infty} \rho_{B \psi}\left(f_{n}-f\right)=0$. Finally, in view of proposition 3.1 , we get $\lim _{n \rightarrow \infty} \rho_{B^{\psi}}\left(f_{n}\right)=\rho_{B^{\psi}}(f)$ and then $F$ is continuous at $k_{0}$. Consequently, since $F(0)=0$ and $\lim _{k \rightarrow \infty} F(k)=+\infty$, there exits $\left.k_{0} \in\right] 0,+\infty[$ for which $\rho_{B^{\psi}}\left[\varphi\left(k_{0}|P|\right)\right]=1$.

Considering the case of equality in the Young's inequality we get :

$$
\begin{aligned}
\|\mid P\|_{B^{\phi}} & \geq \frac{1}{k_{0}} M\left(\left|k_{0} P\right| \cdot \varphi\left(k_{0}|P|\right)\right) \\
& \geq \frac{1}{k_{0}}\left(\rho_{B^{\phi}}\left(k_{0} P\right)+\rho_{B^{\psi}}\left[\varphi\left(k_{0}|P|\right)\right]\right) \\
& \geq \frac{1}{k_{0}}\left(\rho_{B^{\phi}}\left(k_{0} P\right)+1\right)
\end{aligned}
$$

and finally, combining this with (3.5), it follows that

$$
\||P|\|_{B^{\phi}}=\inf _{k>0}\left\{\frac{1}{k}\left(\rho_{B^{\phi}}(k P)+1\right)\right\}=\frac{1}{k_{0}}\left(\rho_{B^{\phi}}\left(k_{0} P\right)+1\right) .
$$

To prove this equality in the general case of $f \in B^{\phi} a . p$., let $\left\{P_{n}\right\}$ be the sequence of Bochner-Fejer's polynomials of the approximation of $f$. From (3.7) we know that, $\left.\forall n \geq 1, \exists k_{n} \in\right] 0,+\infty[$ such that

$$
\left\|\left|P_{n}\right|\right\|_{B^{\phi}}=\left\{\frac{1}{k_{n}}\left(1+\rho_{B^{\phi}}\left(k_{n} P_{n}\right)\right)\right\}
$$

from (3.4) and the properties of the Bochner-Fejer's polynomials (see (1) of 2.2) we get,

$$
\frac{1}{k_{n}} \leq\left\|\left|P_{n}\right|\right\|_{B^{\phi}} \leq 2\left\|P_{n}\right\|_{B^{\phi}} \leq 2\|f\|_{B^{\phi}}
$$

and thus $k_{n} \geq \frac{1}{2\|f\|_{B^{\phi}}}=c_{1}>0$. Let us show that $k_{n} \leq c_{2}, \forall n \geq 0$ for some constant $c_{2}$. Indeed, if this is not the case there exists a subsequence denoted by $\left\{k_{n}\right\}$ increasing to infinity and then

$$
\begin{aligned}
1 & =\rho_{B^{\psi}}\left[\varphi\left(k_{n}\left|P_{n}\right|\right)\right] \\
& \geq \varlimsup_{T \rightarrow+\infty} \frac{1}{2 T} \int_{G_{n}} \psi\left(\varphi\left(k_{n}\left|P_{n}(x)\right|\right)\right) d x \\
& \geq \theta_{1} . \psi\left[\varphi\left(k_{n} \alpha_{1}\right)\right] \underset{n \rightarrow \infty}{\rightarrow} \infty
\end{aligned}
$$


where $G_{n}, \theta_{1}, \alpha_{1}$ are defined in proposition 3.2. A contradiction.

Now, since the sequence $\left\{k_{n}\right\}$ is bounded, there exists a subsequence denoted by $\left\{k_{n}\right\}$ that converge to some $k_{0}$ with $0<k_{0}<+\infty$. Let us show that

$$
\lim _{n \rightarrow \infty} \rho_{B^{\phi}}\left(k_{n} P_{n}\right)=\rho_{B^{\phi}}\left(k_{0} f\right) .
$$

Indeed, we have by (1) of 2.2 ,

$$
\begin{aligned}
\rho_{B^{\phi}}\left(k_{n} P_{n}-k_{0} f\right) & \leq \frac{1}{2} \rho_{B^{\phi}}\left(2\left(k_{n}-k_{0}\right) P_{n}\right)+\frac{1}{2} \rho_{B^{\phi}}\left(2 k_{0}\left(P_{n}-f\right)\right) \\
& \leq\left|k_{n}-k_{0}\right| \rho_{B^{\phi}}(f)+\frac{1}{2} \rho_{B^{\phi}}\left(2 k_{0}\left(P_{n}-f\right)\right)
\end{aligned}
$$

and then $\lim _{n \rightarrow \infty} \rho_{B^{\phi}}\left(k_{n} P_{n}-k_{0} f\right)=0$. Now, in view of proposition 3.1 it follows that $\underline{\lim } \rho_{B^{\phi}}\left(k_{n} P_{n}\right) \geq \rho_{B^{\phi}}\left(k_{0} f\right)$. On the other hand, from the inequality $\rho_{B^{\phi}}\left(\begin{array}{l}n \rightarrow \infty \\ k_{n} P_{n}\end{array}\right) \leq \rho_{B^{\phi}}\left(k_{n} f\right)$, we get,

$$
\varlimsup_{n \rightarrow \infty} \rho_{B^{\phi}}\left(k_{n} P_{n}\right) \leq \varlimsup_{n \rightarrow \infty} \rho_{B^{\phi}}\left(k_{n} f\right)=\lim _{n \rightarrow \infty} \rho_{B^{\phi}}\left(k_{n} f\right)=\rho_{B^{\phi}}\left(k_{0} f\right)
$$

and then,

$$
\begin{aligned}
\varlimsup_{n \rightarrow \infty} \rho_{B^{\phi}}\left(k_{n} P_{n}\right) & \leq \rho_{B^{\phi}}\left(k_{0} f\right) \leq \varliminf_{n \rightarrow \infty} \rho_{B^{\phi}}\left(k_{n} P_{n}\right), \\
\text { i.e. } \lim _{n \rightarrow \infty} \rho_{B^{\phi}}\left(k_{n} P_{n}\right) & =\rho_{B^{\phi}}\left(k_{0} f\right)
\end{aligned}
$$

Finally, letting $n \rightarrow \infty$ in (3.8) it follows

$$
\||f|\|_{B^{\phi}}=\frac{1}{k_{0}}\left(\rho_{B^{\phi}}\left(k_{0} f\right)+1\right)
$$

Consider now the case of a discontinuous $\varphi$. From [8], we know that for each $\varepsilon>0$ there exists an equivalent Orlicz function $\phi_{1}$ with continuous derivative $\varphi_{1}$, more precisely

$$
(1-\varepsilon) \phi(x) \leq \phi_{1}(x) \leq \phi(x), x \geq 0
$$

We have also ( see [3] ) $B^{\phi} a . p .=B^{\phi_{1}} a . p$. as sets and one sees easily that

$$
(1-\varepsilon) \rho_{B^{\phi}}(f) \leq \rho_{B^{\phi_{1}}}(f) \leq \rho_{B^{\phi}}(f), f \in B^{\phi} \text { a.p. }
$$

The same inequality holds for the corresponding norms. 
Now, since (3.9) is true for $\phi_{1}$, using (3.10) we get,

$$
\begin{aligned}
\inf _{k>0}\left\{\frac{1}{k}\left(\rho_{B^{\phi}}(k f)+1\right)\right\} & \leq \inf _{k>0}\left\{\frac{1}{k}\left(\frac{1}{1-\varepsilon} \rho_{B^{\phi_{1}}}(k f)+1\right)\right\} \\
& \leq \frac{1}{1-\varepsilon} \inf _{k>0}\left\{\frac{1}{k}\left(\rho_{B^{\phi_{1}}}(k f)+(1-\varepsilon)\right)\right\} \\
& \leq \frac{1}{1-\varepsilon}\||f|\|_{B^{\phi_{1}}} \\
& \leq \frac{1}{1-\varepsilon}\||f|\|_{B^{\phi}}
\end{aligned}
$$

Finally $\varepsilon>0$ being arbitrary and recalling (3.5), this proves that (3.9) holds in the general case as well.

(2) Suppose first that $\varphi$ is continuous and $f \in\{$ u.a.p. $\}, f \neq 0$. Let $g \in B^{\psi}$ a.p. then,

(i) if $\rho_{B^{\psi}}(g) \leq 1$, we have $M(|f g|) \leq\||f|\|_{B^{\phi}}$.

(ii) if $\rho_{B^{\psi}}(g)>1$, we will have $\rho_{B^{\psi}}\left(\frac{g}{\rho_{B^{\psi}}(g)}\right) \leq \frac{1}{\rho_{B^{\psi}}(g)} \rho_{B^{\psi}}(g)=1$ and then $M\left(\left|f \frac{g}{\rho_{B^{\psi}}(g)}\right|\right) \leq\||f|\|_{B^{\phi}}$.

It follows that in all cases we have,

$$
M(|f g|) \leq \max \left(1, \rho_{B^{\psi}}(g)\right) \cdot\||f|\|_{B^{\phi}} .
$$

Suppose now that $g=\varphi\left(\frac{f}{\||f|\|_{B^{\phi}}}\right)$, then $g \in\{$ u.a.p. $\}$ and using the case of equality in the Young's inequality and the fact that in this case the limit exists, we may write

$$
M\left(\left|\frac{f}{\||f|\|_{B^{\phi}}} g\right|\right)=\rho_{B^{\phi}}\left(\frac{f}{\||f|\|_{B^{\phi}}}\right)+\rho_{B^{\psi}}(g) \leq \max \left(1, \rho_{B^{\psi}}(g)\right)
$$

so that we get $\rho_{B^{\phi}}\left(\frac{f}{\||f|\|_{B^{\phi}}}\right) \leq 1$.

To consider the general case of $f \in B^{\phi}$ a.p., let $\left\{P_{n}\right\}$ be the sequence of BochnerFejer's polynomials of the approximation of $f$, we have :

$$
\rho_{B^{\phi}}\left(\frac{P_{n}}{\left\|\left|P_{n}\right|\right\|_{B^{\phi}}}\right) \leq 1, \forall n \geq 1
$$

But, in view of proposition 3.1 and (1) of 2.2 , we can write :

$$
\left\|\left|P_{n}\right|\right\|_{B^{\phi}}=\inf _{k>0}\left\{\frac{1}{k}\left(1+\rho_{B^{\phi}}\left(k P_{n}\right)\right)\right\} \leq \inf _{k>0}\left\{\frac{1}{k}\left(1+\rho_{B^{\phi}}(k f)\right)\right\}=\||f|\|_{B^{\phi}}
$$


so that,

$$
\rho_{B^{\phi}}\left(\frac{P_{n}}{\||f|\|_{B^{\phi}}}\right) \leq \rho_{B^{\phi}}\left(\frac{P_{n}}{\left\|\left|P_{n}\right|\right\|_{B^{\phi}}}\right) \leq 1,
$$

and then by (3) of proposition 3.1 it follows $\rho_{B^{\phi}}\left(\frac{f}{\||f|\|_{B^{\phi}}}\right) \leq 1$.

In the genaral case of a discontinuous $\varphi$, we use the inequalities (3.10) to obtain

$$
\rho_{B^{\phi}}\left(\frac{f}{\||f|\|_{B^{\phi}}}\right) \leq \rho_{B^{\phi}}\left(\frac{f}{\||f|\|_{B^{\phi_{1}}}}\right) \leq \rho_{B^{\phi_{1}}}\left(\frac{f}{(1-\varepsilon)\||f|\|_{B^{\phi_{1}}}}\right)
$$

Finally, using lemma 3.3 we get

$$
\rho_{B^{\phi}}\left(\frac{f}{\||f|\|_{B^{\phi}}}\right) \leq \lim _{\varepsilon \rightarrow 0} \rho_{B^{\phi_{1}}}\left(\frac{f}{(1-\varepsilon)\||f|\|_{B^{\phi_{1}}}}\right)=\rho_{B^{\phi_{1}}}\left(\frac{f}{\||f|\|_{B^{\phi_{1}}}}\right) \leq 1
$$

(3) We have, $\rho_{B^{\phi}}\left(\frac{f}{\||f|\|_{B^{\phi}}}\right) \leq 1$ and then $\|f\|_{B^{\phi}} \leq\||f|\|_{B^{\phi}}$. Finally, in view of (3.4) we get,

$$
\|f\|_{B^{\phi}} \leq\||f|\|_{B^{\phi}} \leq 2\|f\|_{B^{\phi}} .
$$

Proposition 3.5. Let $f \in E^{\phi}([0,1])$ where $E^{\phi}([0,1])$ is the Orlicz class of functions

$$
E^{\phi}([0,1])=\left\{f \text { measurable s.t. } \rho_{\phi}(\lambda f)<+\infty, \forall \lambda>0\right\},
$$

where $\rho_{\phi}(f)=\int_{0}^{1} \phi(|f|) d \mu$ is the usual Orlicz modular. Then,

1. If $\widetilde{f}$ is the periodic extension of $f$ to the whole $\mathbb{R}$ ( with period $\tau=1$ ), we have $\tilde{f} \in B^{\phi}$ a.p..

2. The injection $i: E^{\phi}([0,1]) \rightarrow B^{\phi}$ a.p., $i(f)=\tilde{f}$ is an isometry with respect to the modulars and for the respective Orlicz norms.

\section{Proof.}

1. Let $f=\sum_{i=1}^{n} a_{i} \chi_{A_{i}}, A_{i} \cap A_{j}=\emptyset$ if $i \neq j$ and $\bigcup_{i=1}^{n} A_{i} \subset[0, \alpha], 0<\alpha<1$. Let $m \in \mathbb{N} \backslash\{0\}$, since $\sum_{i=1}^{n} \phi\left(m a_{i}\right) \mu\left(A_{i}\right)<+\infty$, it follows from (3.1) that there exists $P_{m} \in \mathcal{P}$ ( the set of generalized trigonometric polynomials ) for which $\rho_{B^{\phi}}\left(\frac{m}{4}\left(\tilde{f}-P_{m}\right)\right) \leq \frac{1}{m}$ where $\widetilde{f}$ is the periodic extension ( of period $\tau=1$ ) 
of $f$ to the whole $\mathbb{R}$.

Let $\lambda>0$ be given and $m_{0} \in \mathbb{N} \backslash\{0\}$ such that $\lambda \leq \frac{m_{0}}{4}$ then,

$$
\rho_{B^{\phi}}\left(\lambda\left(\tilde{f}-P_{m}\right)\right) \leq \rho_{B^{\phi}}\left(\frac{m}{4}\left(\tilde{f}-P_{m}\right)\right) \leq \frac{1}{m}, \forall m \geq m_{0} .
$$

This means that $\lim _{m \rightarrow+\infty}\left\|\tilde{f}-P_{m}\right\|_{B^{\phi}}=0$, i.e. $\tilde{f} \in B^{\phi} a . p$.

Consider now the general case of $f \in E^{\phi}([0,1])$. It is known (see [2]) that the step functions are dense in $E^{\phi}([0,1])$, hence given $\varepsilon>0$, there is a $g_{\varepsilon}=\sum_{i=1}^{n} a_{i} \chi_{A_{i}}$ for which $\left\|g_{\varepsilon}-f\right\|_{\phi} \leq \frac{\varepsilon}{4}$. Since $f$ is absolutely continuous, we may choose $\delta>0$ such that $\mu(A) \leq \delta \Rightarrow\left\|f \chi_{A}\right\|_{\phi} \leq \frac{\varepsilon}{4}$. We take $\alpha>0$ with $1-\alpha \leq \delta$ and put $A_{i}^{\alpha}=A_{i} \cap[0, \alpha], i=1, n$. Let $g_{\varepsilon}^{\alpha}=\sum_{i=1}^{n} a_{i} \chi_{A_{i}^{\alpha}}$ then $g_{\varepsilon}^{\alpha} \in E^{\phi}([0,1])$. If $\tilde{f}$ and $\widetilde{g}_{\varepsilon}^{\alpha}$ are the respective periodic extensions ( with period $\tau=1$ ) of $f$ and $g_{\varepsilon}^{\alpha}$, we will have,

$$
\begin{aligned}
\left\|\tilde{f}-\widetilde{g}_{\varepsilon}^{\alpha}\right\|_{B^{\phi}} & =\left\|f-g_{\varepsilon}^{\alpha}\right\|_{\phi} \\
& \leq\left\|\left(f-g_{\varepsilon}^{\alpha}\right) \chi_{[0, \alpha]}\right\|_{\phi}+\left\|\left(f-g_{\varepsilon}^{\alpha}\right) \chi_{[\alpha, 1]}\right\|_{\phi} \\
& \leq\left\|f-g_{\varepsilon}\right\|_{\phi}+\left\|f \chi_{[\alpha, 1]}\right\|_{\phi} \\
& \leq \frac{\varepsilon}{4}+\frac{\varepsilon}{4}=\frac{\varepsilon}{2}
\end{aligned}
$$

Now, since $\widetilde{g}_{\varepsilon}^{\alpha} \in B^{\phi}$ a.p. there exists $P_{\varepsilon} \in \mathcal{P}$ for which $\left\|\widetilde{g}_{\varepsilon}^{\alpha}-P_{\varepsilon}\right\|_{B^{\phi}} \leq \frac{\varepsilon}{2}$.

Finally,

$$
\left\|\widetilde{f}-P_{\varepsilon}\right\|_{B^{\phi}} \leq\left\|\widetilde{f}-\widetilde{g}_{\varepsilon}^{\alpha}\right\|_{B^{\phi}}+\left\|\widetilde{g}_{\varepsilon}^{\alpha}-P_{\varepsilon}\right\|_{B^{\phi}} \leq \frac{\varepsilon}{2}+\frac{\varepsilon}{2}=\varepsilon .
$$

i.e. $\tilde{f} \in B^{\phi}$ a.p.

2. It is clear that $i: E^{\phi}([0,1]) \rightarrow B^{\phi}$ a.p. is a modular isometry.

The fact that it is also an isometry for the Orlicz norms follows immediately since

$$
\||f|\|_{\phi}=\inf _{k>0}\left\{\frac{1}{k}\left(1+\rho_{\phi}(k f)\right)\right\}=\inf _{k>0}\left\{\frac{1}{k}\left(1+\rho_{B^{\phi}}(k \widetilde{f})\right)\right\}=\|\mid \tilde{f}\|_{B^{\phi}}
$$




\section{Strict convexity of the space $B^{\phi}$ a.p.}

Now, we can characterize the strict convexity of the space $B^{\phi}$ a.p..

Theorem 4.1. The space $B^{\phi}$ a.p. induced with the Orlicz norm is strictly convex if and only if the function $\phi$ is strictly convex on $\mathbb{R}$.

Proof. Sufficiency. Let $f, g \in B^{\phi}$ a.p. be such that $\||f|\|_{B^{\phi}}=\||g|\|_{B^{\phi}}=1$ and $\||f-g|\|_{B^{\phi}}>0$. For $s \in K(f)$ and $m \in K(g)$ we have $\||s f-m g|\|_{B^{\phi}}>0$, since in the contrary case we will get $\||s f|\|_{B^{\phi}}=\||m g|\|_{B^{\phi}}$ and then $s=m$ so that $\||f-g|\|_{B^{\phi}}=0$, a contradiction.

Now by proposition 3.2 there exist $\alpha>0$ and $\theta \in(0,1)$ such that for the set $G=\{t \in \mathbb{R},|s f(t)-m g(t)| \geq \alpha\}$ we have $\bar{\mu}(G)>\theta$. It follows from this,

$$
\rho_{B^{\phi}}(s f-m g) \geq \phi(\alpha) \cdot \bar{\mu}(G) \geq \phi(\alpha) \cdot \theta>0 .
$$

Let $k>1$ be such that $\bar{\mu}(A) \geq \frac{\theta}{4} \Rightarrow\left\|\chi_{A}\right\|_{B^{\phi}}>\frac{1}{k}$ and define the sets,

$$
A_{1}=\{t \in \mathbb{R},|f(t)| \geq k\} \quad ; \quad A_{2}=\{t \in \mathbb{R},|g(t)| \geq k\}
$$

we have,

$$
1=\||f|\|_{B^{\phi}} \geq\|f\|_{B^{\phi}} \geq\left\|f \chi_{A_{1}}\right\|_{B^{\phi}} \geq k\left\|\chi_{A_{1}}\right\|_{B^{\phi}}
$$

i.e. $\left\|\chi_{A_{1}}\right\|_{B^{\phi}} \leq \frac{1}{k}$ and then $\bar{\mu}\left(A_{1}\right)<\frac{\theta}{4}$. By similar computations we get also $\bar{\mu}\left(A_{2}\right)<$ $\frac{\theta}{4}$.

From the strict convexity of $\phi$, there exists $\delta>0$ such that,

$$
\phi(r u+(1-r) v) \leq(1-\delta)[r \phi(u)+(1-r) \phi(v)]
$$

for each $r \in\left[\frac{1}{1+b}, \frac{b}{b+1}\right]$ and $|u| \leq b k,|v| \leq b k,|u-v| \geq r$ where $b=\max (s, m)$.

Since $\frac{s}{s+m}$ and $\frac{m}{s+m}$ are in $\left[\frac{1}{1+b}, \frac{b}{b+1}\right]$, we will have for $t \in G \backslash\left(A_{1} \cup A_{2}\right)$,

$$
\phi\left(\frac{s m}{s+m}(f(t)+g(t))\right) \leq(1-\delta)\left[\frac{s}{s+m} \phi(m f(t))+\frac{m}{s+m} \phi(s g(t))\right]
$$


Then using the results in proposition 3.4 it follows,

$$
\begin{aligned}
& 2-\||f+g|\|_{B^{\phi}} \\
& \geq \frac{1}{s}\left(1+\rho_{B^{\phi}}(s f)\right)+\frac{1}{m}\left(1+\rho_{B^{\phi}}(m g)\right)-\frac{s+m}{s m}\left(1+\rho_{B^{\phi}}\left(\frac{s m}{s+m}(f+g)\right)\right) \\
& \geq \lim _{T \rightarrow+\infty} \frac{1}{2 T} \int_{-T}^{+T}\left[\frac{m}{s+m} \phi(s f(t))+\frac{s}{s+m} \phi(m g(t))-\phi\left(\frac{s m}{s+m}(f(t)+g(t))\right)\right] d t \\
& \geq \lim _{T \rightarrow+\infty} \frac{1}{2 T} \int_{-T}^{+T}\left[\frac{\delta}{s} \phi(s f(t))+\frac{\delta}{m} \phi(m g(t))\right] d t \\
& \geq \frac{2 \delta}{b} \lim _{T \rightarrow+\infty} \frac{1}{2 T} \int_{-T}^{+T}\left[\frac{\phi(s f(t))+\phi(-m g(t))}{2}\right] d t \\
& \geq \frac{2 \delta}{b} \lim _{T \rightarrow+\infty} \frac{1}{2 T} \int_{-T}^{+T}\left[\phi\left(\frac{|s f(t)-m g(t)|}{2}\right)\right] d t \\
& \geq \frac{2 \delta}{b} \lim _{T \rightarrow+\infty} \frac{1}{2 T} \int_{\left(G \backslash\left(A_{1} \cup A_{2}\right)\right) \cap[-T, T]}\left[\phi\left(\frac{|s f(t)-m g(t)|}{2}\right)\right] d t \\
& \geq \frac{2 \delta}{b} \phi\left(\frac{\alpha}{2}\right) \bar{\mu}\left(G \backslash\left(A_{1} \cup A_{2}\right)\right) \geq \frac{\delta}{b} \phi\left(\frac{\alpha}{2}\right) \cdot \theta>0 .
\end{aligned}
$$

and then, $B^{\phi}$ a.p. is strictly convex.

Necessity: The strict convexity of $\phi$ is necessary for the strict convexity of the Orlicz class $E^{\phi}([0,1])(c . f .[2])$. Using proposition 3.5, we deduce that is also necessary for the strict convexity of $B^{\phi} a . p$. .

\section{References}

[1] Besicovitch A.S., Almost Periodic Functions, Dover Pub.Inc.,NewYork (1954).

[2] Chen, S., Geometry of Orlicz spaces. Dissertationes Math. No 356 (1996).

[3] Hillmann T.R., Besicovitch-Orlicz spaces of almost periodic functions, Real and Stochastic Analysis, Wiley, 1986, pp. 119-167.

[4] Krasnoselskii M.A. and Rutickii Ya.B., Convex function and Orlicz spaces, P.Noodhoff Ltd.,Groningen,(1961).

[5] Morsli M., On some convexity properties of the Besicovitch-Orlicz space of almost periodic functions, Comment.Math. 34 (1994), 137-152.

[6] Morsli M., Espace de Besicovitch Orlicz de fonctions presque periodiques. Structure generale et geometrie, These de Doctorat (1996). 
[7] Morsli M., On modular approximation property in the Besicovitch-Orlicz space of almost periodic functions, Comment. Math. Univ. Carolinae 38,3 (1997), 485-496.

[8] Rao M.M., Ren Z.D., Theory of Orlicz spaces, Marcel Dekker, Inc. New-York, (1991). 